\title{
Experimental Study of High-Z Gas Buffers in Gas-Filled ICF Engines
}

M. A. Rhodes, J. Kane, G. Loosmore, J. DeMuth, J. Latkowski

December 14, 2010

2011 Photonics West Advance Technical Program San Francisco, CA, United States January 22, 2011 through January 27, 2011 
This document was prepared as an account of work sponsored by an agency of the United States government. Neither the United States government nor Lawrence Livermore National Security, LLC, nor any of their employees makes any warranty, expressed or implied, or assumes any legal liability or responsibility for the accuracy, completeness, or usefulness of any information, apparatus, product, or process disclosed, or represents that its use would not infringe privately owned rights. Reference herein to any specific commercial product, process, or service by trade name, trademark, manufacturer, or otherwise does not necessarily constitute or imply its endorsement, recommendation, or favoring by the United States government or Lawrence Livermore National Security, LLC. The views and opinions of authors expressed herein do not necessarily state or reflect those of the United States government or Lawrence Livermore National Security, LLC, and shall not be used for advertising or product endorsement purposes. 


\title{
Experimental Study of High-Z Gas Buffers in Gas-Filled ICF Engines
}

\author{
M.A. Rhodes, J. Kane, G. Loosmore, J. DeMuth, J. Latkowski \\ Lawrence Livermore National Laboratory, PO Box 808 L-460, Livermore CA
}

\begin{abstract}
ICF power plants, such as the LIFE scheme at LLNL, may employ a high-Z, target-chamber gas-fill to moderate the first-wall heat-pulse due to x-rays and energetic ions released during target detonation.

To reduce the uncertainties of cooling and beam/target propagation through such gas-filled chambers, we present a pulsed plasma source producing 2-5 eV plasma comprised of high-Z gases. We use a 5-kJ, 100-ns theta discharge for high peak plasma-heating-power, an electrode-less discharge for minimizing impurities, and unobstructed axial access for diagnostics and beam (and/or target) propagation studies. We will report on the plasma source requirements, design process, and the system design.
\end{abstract}

Keywords: Xenon plasma, inertial fusion energy, theta pinch, first wall protection

\section{INTRODUCTION}

Power plants driven by nuclear fusion energy use either magnetic or inertial plasma confinement to achieve the temperature and density required for net energy output. In inertial confinement schemes, one of several types of highenergy beams (laser, ion, or electron) impinge on a target containing fusion fuel. The beam-energy heats and compresses the target to thermonuclear conditions. The energy released during the thermonuclear burn is then converted in the power plant to electricity.

In the Laser Inertial Fusion Energy (LIFE) $)^{1}$ scheme, currently under development at Lawrence Livermore National Laboratory, fusion targets are injected into the central fusion engine. Pulsed laser beams are concurrently injected into the fusion engine and impinge on the targets roughly at the engine's center. Energy is released from the target in three forms: fusion-neutrons, $\mathrm{x}$-rays, and energetic ions. The fusion-neutrons pass through the fusion-engine first-wall without significant absorption. However, the $\mathrm{x}$-rays and energetic ions would be entirely absorbed by the first-wall without some form of mitigation. This would lead to first-wall overheating and damage. The LIFE scheme uses a high- $Z$ gas-fill (nominally xenon at a pressure near 1 Torr) to delay the x-ray and ion flux to the first-wall. This controls the first-wall temperature pulse but also results in heating and ionization of the gas-fill. The heated and ionized gas can then adversely affect targets and laser pulses injected for subsequent shots. For example, the injected laser energy can be absorbed in the ionized gas by inverse Bremsstraulung. The targets, which may contain cryogenic fusion fuel, might absorb too much heat from the gas-fill during the injection process vaporizing the frozen fuel.

Therefore, it is important to understand the rate at which such a xenon gas-fill recombines and cools. Modeling of xenon gas fills with radiation hydrodynamic codes such as Hydra, suggest that the gas near the target will be rapidly $(<1 \mathrm{~ns})$ heated to high temperature $(>10-\mathrm{eV})$ and high ionization states $(>10)$. The modeling also suggests that within several hundred nanoseconds this hot xenon plasma will cool to $<2-\mathrm{eV}$ but the cooling then stalls because xenon ceases to radiate. However, the atomic physics models used by codes like Hydra are known to be inaccurate below 10-eV and very inaccurate below 1-eV. Hence, the final state of the gas-fill, based on modeling, after the 70-ms between shots is very uncertain.

To reduce this uncertainty, we are designing and building a xenon plasma source so we can measure the actual xenon cooling rates and measure the effect of the heated xenon on laser pulses and injected targets. In this paper, we will discuss the plasma the source requirements and design process in Section 2. We will present the experimental design now under construction in Section 3 and conclude in Section 4. 


\section{PLASMA SOURCE REQUIRMENTS AND DESIGN PROCESS}

We want to design a plasma source that will reproduce the plasma conditions expected in the LIFE xenon gas-fill. We can state this requirement in terms of the density required and the temperature required. Since the LIFE gas-fill pressure is expected to be in the range of $0.5-1.0$-Torr, this leads to a density in the $10^{16}$ particles $/ \mathrm{cm}^{3}$ range. The required temperature is a little more difficult to define. In order to perform a meaningful cooling experiment, we must first heat the xenon to a temperature higher than a starting point of interest. This is nominally $10-\mathrm{eV}$ since above $10-\mathrm{eV}$ we have high confidence in our codes. We'll see below that $10-\mathrm{eV}$ is probably too difficult to achieve and we'll have to settle for an initial heated temperature of $2-3-\mathrm{eV}$. This will be hot enough to form a fully ionized plasma with an average ionization state (Z-bar) $>1$.

There is also a requirement on the plasma source duration. We would like our source to heat the entire gas-fill and not just the electrons However, plasma sources tend to initially deposit their input energy into ionization and electron heating. The ions or neutrals are only heated by collisions with the electrons. In the density range of interest $\left(10^{16} \mathrm{~cm}^{-3}\right)$, the electron to ion thermal equilibration time is on the order of 100-ns. Our plasma source must therefore heat the electrons to the desired temperature and maintain this temperature long enough to allow equilibration with the ions and neutrals.

No matter what type of plasma source we choose, the electron temperature achieved will be an equilibrium between the heating power-in and the radiation power-out. We have used the 1-D, commercial, radiation hydrodynamic code Hyades $^{2}$ to estimate the resulting electron temperature as a function of input power. The model is a 1-D cylindrical slug (1-cm long by definition) and we can choose the inner and out radius. We keep hydrodynamic motion off and use the average atom model for ionization and radiation. We use an artificial electron heat source that adds energy to the electrons as a constant power source. Figure 1 shows the equilibrium temperature as a function of input power after 500ns of simulation time for two different cylindrical diameters. The 4-cm diameter case runs cooler than the 2-cm diameter case because there is more mass and more outer surface area for radiation loss.

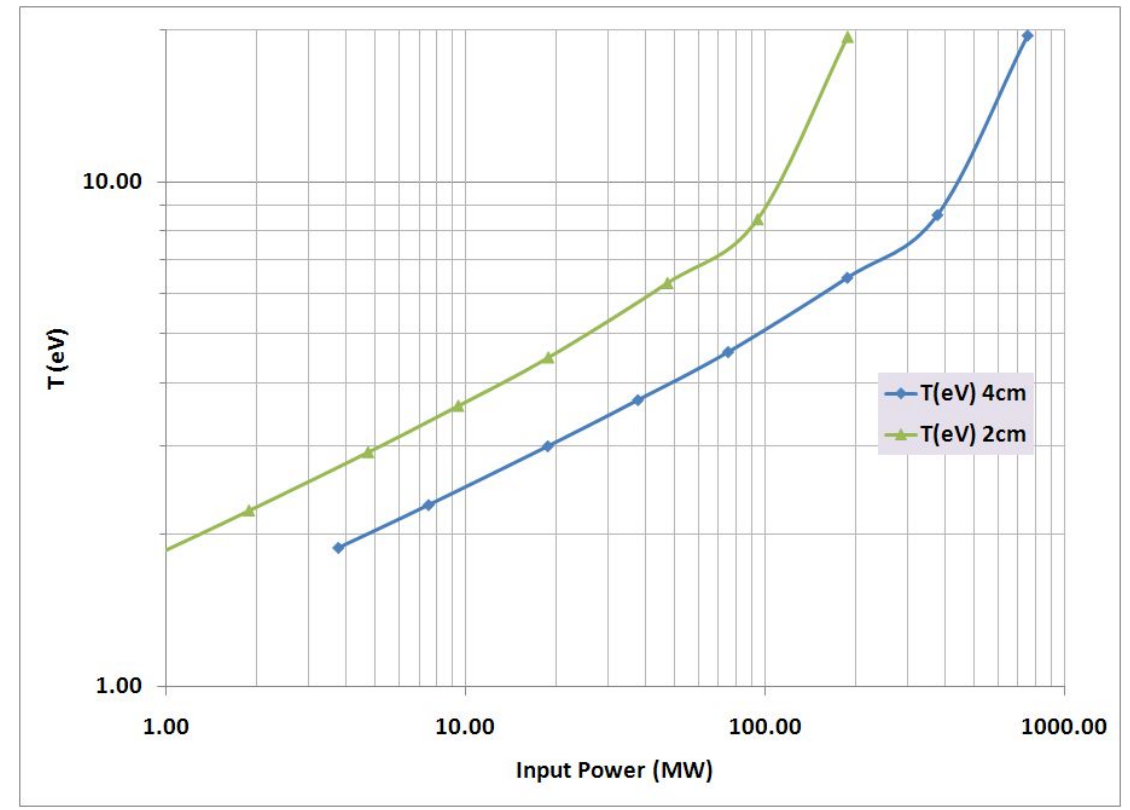

Figure 1. Hyades results showing equilibrium plasma temperature as a function of electron heating power after 500-ns of simulation time for two different diameter cylindrical section (1-cm deep).

We can use these results to design the plasma source. We'll assume that the input power will be from electrically driven Ohmic heating so we need to determine the current required for a desired power input. We use the plasma conditions from the Hyades results to determine the Spitzer resistivity for each case. Then, using the mean plasma perimeter for a given plasma size we can determine the resistance of the plasma. Finally, from $\mathrm{P}=\mathrm{I}^{2} \mathrm{R}$, we can determine the current required to achieve that power input. This gives the current required per linear centimeter of plasma. A plot of plasma 
temperature as a function of current/cm is shown in Figure 2. A current of $10-\mathrm{kA} / \mathrm{cm}$ should give us a plasma temperature between $2-3 \mathrm{eV}$ while $100-\mathrm{kA}$ per cm would be needed to heat the plasma to $10-\mathrm{eV}$.

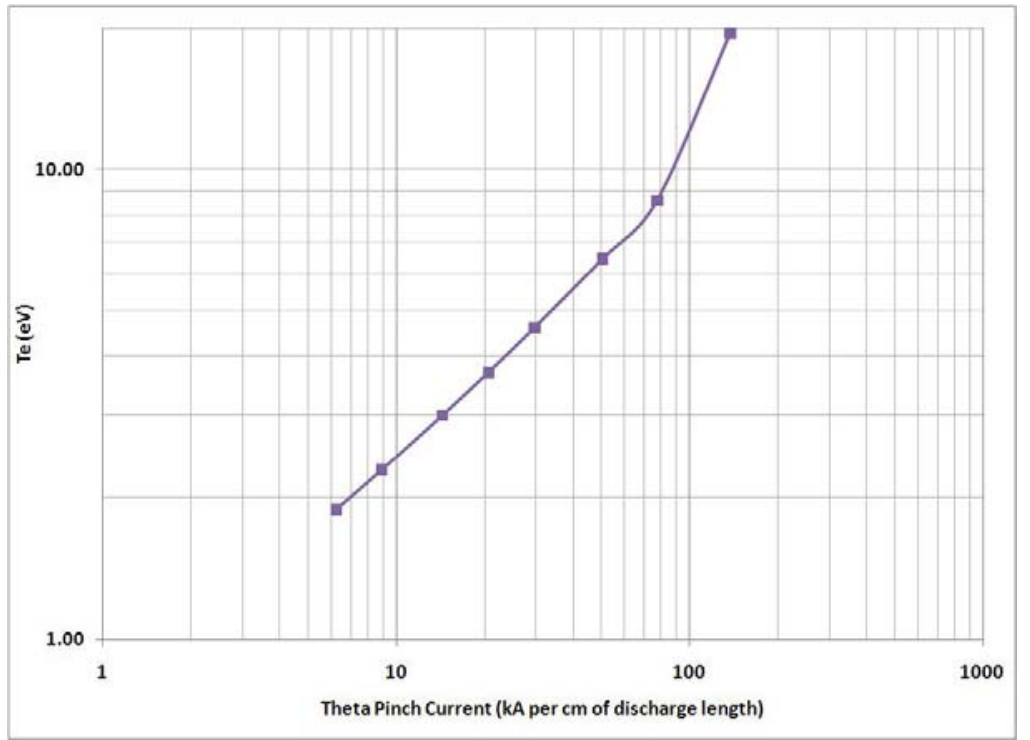

Figure 2. Predicted plasma temperature as a function of azimuthal current using the Hyades radiation power balance results.

Now we need to means to drive the required current in a practical device. The model described above assumes an azimuthal current in a cylindrical section. This can be achieved by using a theta-pinch geometry as shown in Figure 3. We use a capacitor discharge system to drive a current pulse in a cylindrical coil of one or more turns surrounding a gasfilled glass tube. Current is then induced in the plasma by the current pulse in the coil.

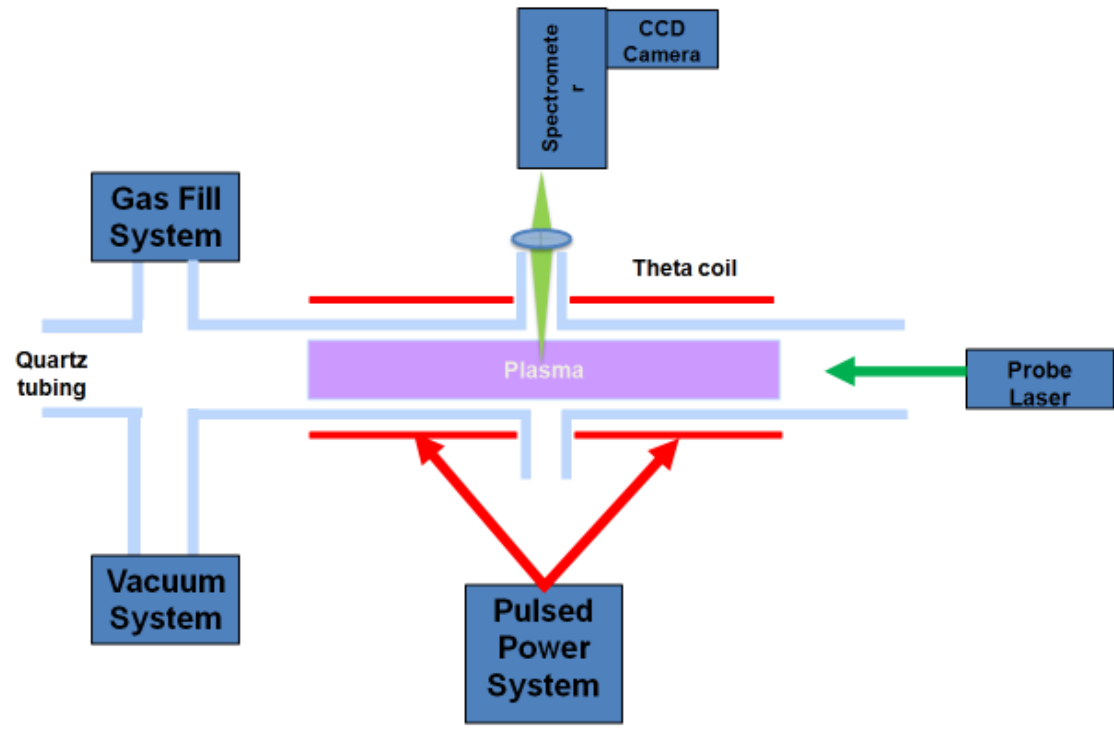

Figure 3. Diagram of our experimental concept to drive an induced azimuthal current in the plasma

We have used the commercial code Maxwell ${ }^{3}$ to compute the inductance of various coil designs, the inductance of the plasma, and the mutual inductance (coupling coefficient) between a coil and the plasma. We then use these results to build a circuit model of the entire electrical system. A typical circuit is shown in Figure 4. We simulate the response of this circuit with yet another commercial code, PSPICE ${ }^{4}$. A typical result is shown in Figure 5 where we can see the coil current compared to the induced plasma current for two different coil designs. 


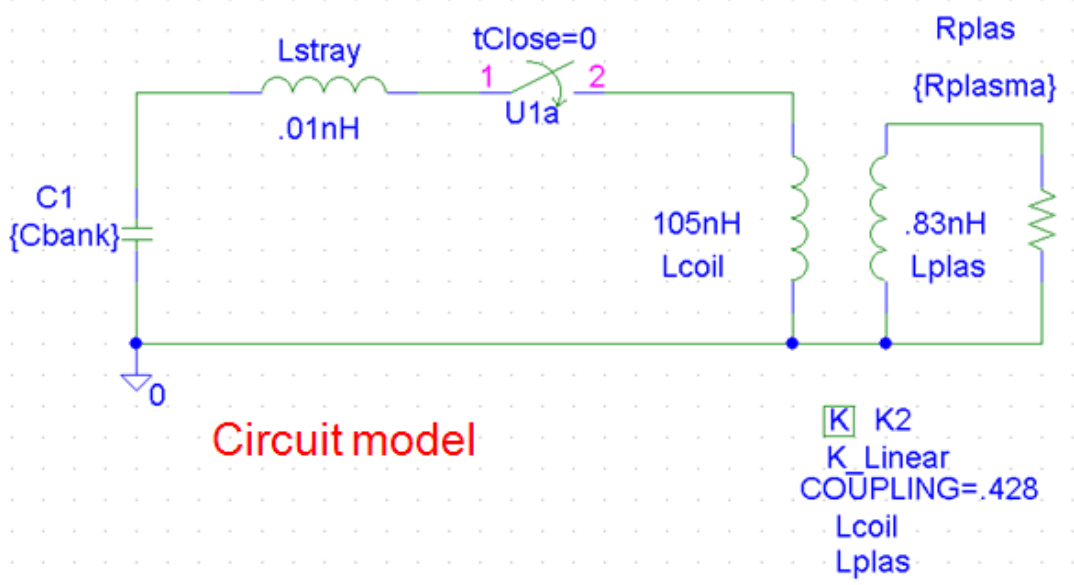

Figure 4. Typical circuit model for simulating the plasma current. Inductance values $\left(\mathrm{L}_{\text {coil }}, \mathrm{L}_{\text {plas }}\right.$, and $\left.\mathrm{k}\right)$ are computed with Ansoft Maxwell.

We have used this process to look for an optimum coil design based on the number of turns. The waveforms in Figure 5 show the results for one-turn and four-turn coils. We can see that the four-turn coil results in higher plasma current with less coil current. This optimum results from the unavoidable finite inductance of the drive circuit. If one could build a drive circuit with zero inductance, the optimum coil would always be one-turn. However, in real circuits, it is difficult to design a system where the circuit stray inductance is small compared to the one-turn coil inductance.

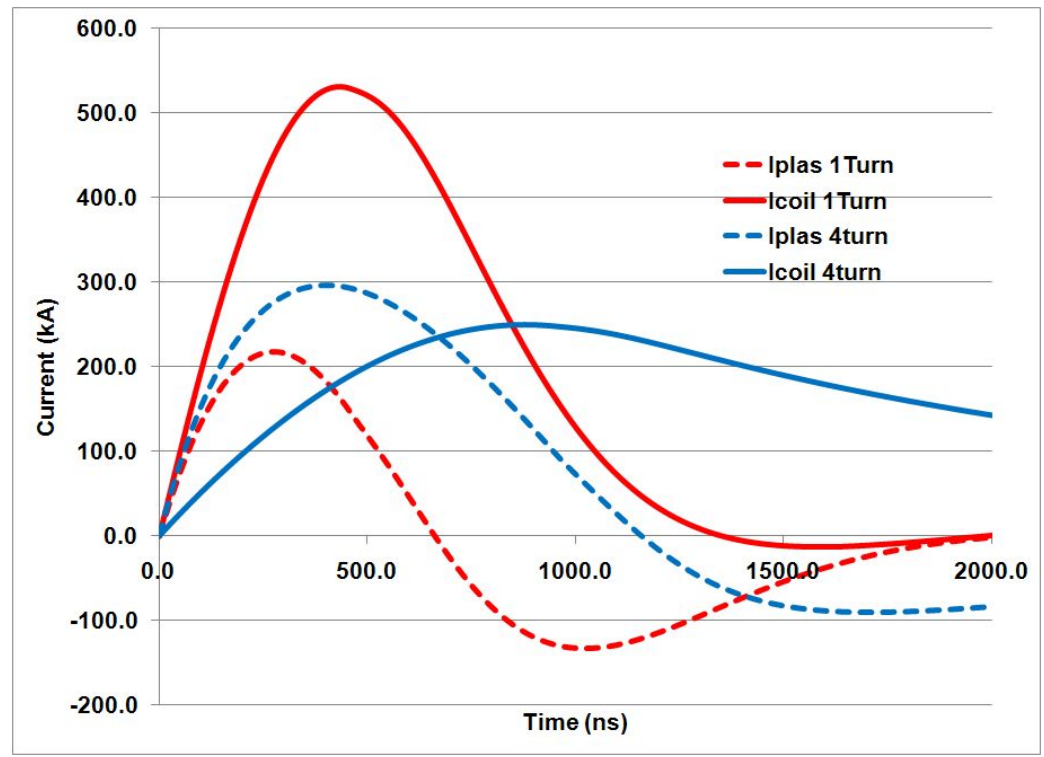

Figure 5. PSPICE simulation of coil and plasma currents for a 1-turn coil and a 4-turn coil. The 4-turn coil produces about $50 \%$ more induced plasma current with half the coil current.

The final step in the design process is to check the self-induced magnetic force on the coil. If the magnetic force in the coil for the desired current destroys the coil, it's not a practical design. To compute the magnetic force, we start with the eddy current solver in Maxwell. We choose a solution frequency of $2.5 \mathrm{MHz}$, which approximates the temporal evolution of the planned current pulse. The result of this simulation yields the current density in the coil including the AC skin-depth effects. Next, we use the Maxwell post-processor to compute the JxB force-density field inside the coil. We then use this force-density field as an external load in Ansys Mechanical ${ }^{5}$ to get the coil stress and displacement. We performed this calculation with a relatively low coil current $(10-\mathrm{kA})$ to keep the mechanical response linear. Figure 6 shows the results for our base-line coil design. Figure 6a is the Von Mises coil stress and Figure 6b is the coil displacement. The displacements are exaggerated in the plot by a large factor too make them easier to visualize. We can 
scale this result to the higher peak currents of interest by assuming that the stress and displacement scale with $\mathrm{I}^{2}$. Based on this scaling, the coil shown will be mechanically adequate up to about 85-kA. To operate at higher current, will need to use some form of reinforced coil design.
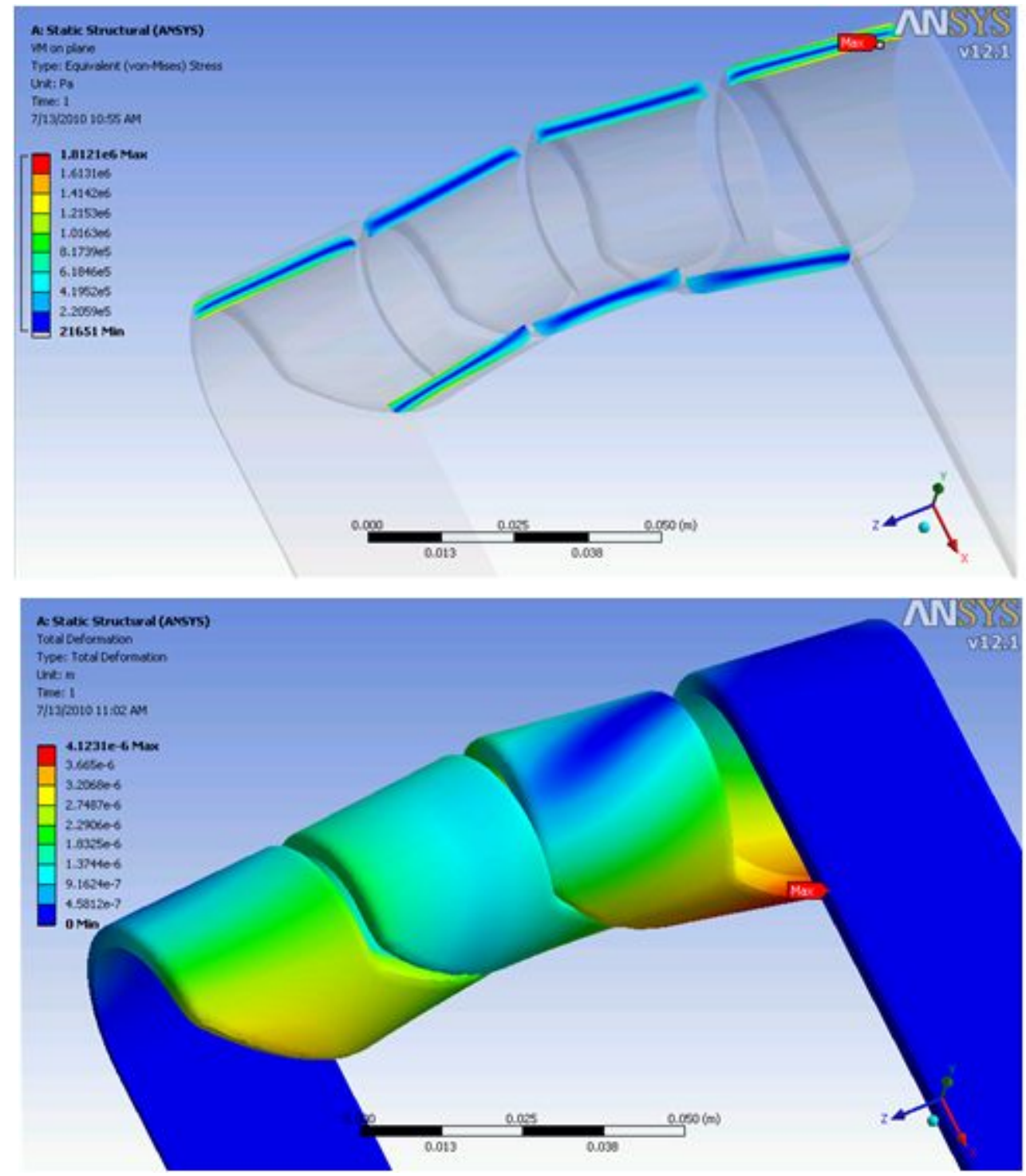

Figure 6. ANSYS Mechanical simulations of coil stress and deformation from self-induced magnetic forces for 10-kA of coil current. a) Von Mises stress shown on a cut plane through the coil. b) deformation of the coil.

The design process detailed above is not self consistent and assumes uniform current distribution in the cylindrical plasma. More detailed and self consistent modeling with Hydra is presented in a companion paper. ${ }^{6}$

\section{PLASMA SOURCE DESIGN}

Our xenon plasma source, as designed, is shown in Figure 7. The pulsed power system consists of four plastic-housing energy storage capacitors each rated at $0.4-\mu \mathrm{F}$ at $100-\mathrm{kV}$. The charged capacitors are switched to the coil-load by a pair of series spark-gap switches. A second pair of shunt spark-gap switches provides a crowbar function to minimize voltage reversal on the capacitors. The current pulse connects to the drive coil by a plate transmission line. While the system is designed to use coils of various designs, our baseline coil is a center-fed, split coil design. Each half-coil consists of four turns. These two half-coils are electrically in parallel and slip over a glass or quartz tube. This design is electrically 
equivalent to a four-turn coil but has better symmetry, lower connection inductance, and allows central ports that would be difficult to implement with a continuous four-turn design.

The entire plasma source is contained within a stainless steel tank. The tank is both gas and liquid tight. We have designed the system for initial operation insulated only by ambient air in the tank. This will allow operation at charging voltage up to about $40-\mathrm{kV}$. This will be enough voltage to get initial results and validate our diagnostics. For operation at higher voltage, we will need to fill the tank with either gaseous $\mathrm{SF}_{6}$ or transformer oil. While the capacitors are rated at $100-\mathrm{kV}$, the existing switches are rated at $75-\mathrm{kV}$. Operation at the full $100-\mathrm{kV}$ would require modified switches or switches with a higher rating.

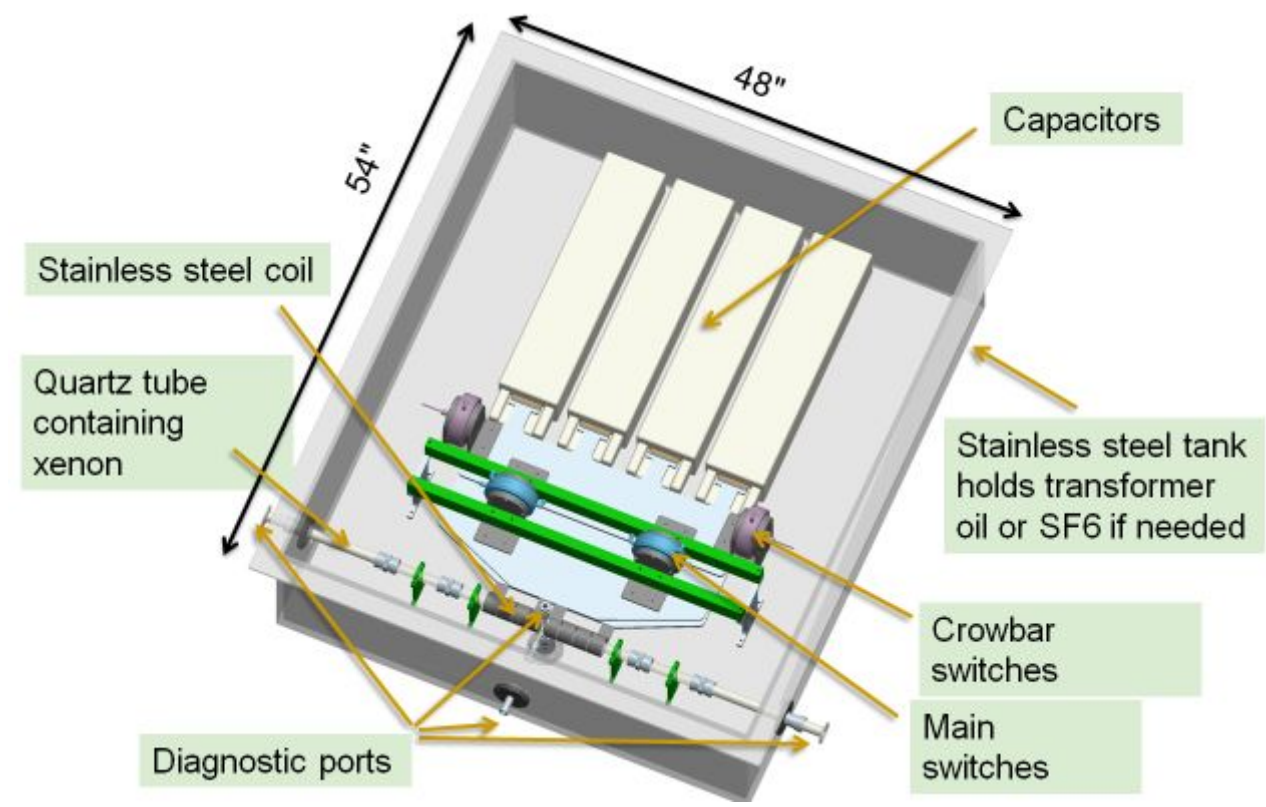

Figure 7. Design of the plasma source

A diagram of the experimental layout is shown in Figure 8. The experiment is built on four vibration-isolated tables. One table supports the experimental source. A second table supports a diagnostic laser. A third table supports a laser beam dump and any diagnostics required for light transmission experiments. A fourth table supports a spectrometer and CCD camera system.

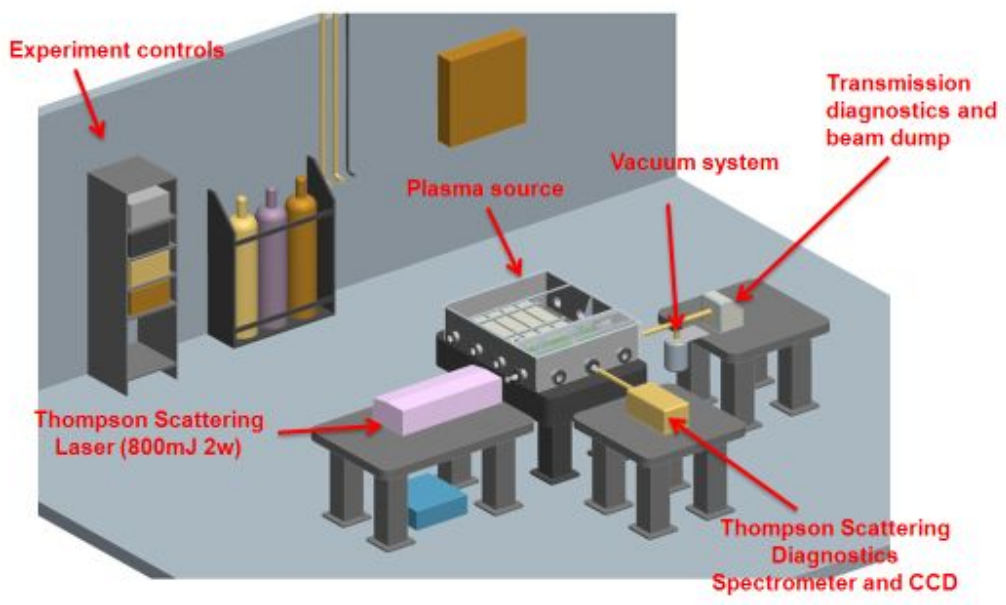

Figure 8. Laboratory layout of our plasma source 
We will use a variety of diagnostics to measure the plasma formed in this device. Our primary diagnostic for electron temperature and density will be a Thomson scattering system based on an 800-mJ, 532-nm, Nd YAG laser along with a .75-meter spectrometer and a gated, intensified, CCD camera-system. We will also use Langmuir probes and spectroscopy diagnostics to obtain further information about the plasma. The Langmuir probes will be particularly useful to measure the longer term decay and cooling of the plasma after the plasma formation pulse. The Thomson scattering system will be used primarily to determine the peak electron temperature during the current pulse.

\section{CONCLUSION}

The baseline design for the LIFE laser fusion power plant employs a gas-filled core to mitigate the first-wall heat pulse. Energy released by the fusion targets will heat and ionize this gas which could lead to absorption of subsequent laser pulses and/or excessive heating of injected targets. While gas-fill modeling with radiation hydrodynamic codes is very useful, such codes are known to be inaccurate at low plasma temperature $(<1-\mathrm{eV})$ where the plasma is transitioning to hot gas. To reduce the uncertainty of the gas-fill conditions at millisecond timescales, we have designed a pulsed plasma source that will produce xenon plasma with a temperature $>2 \mathrm{eV}$. We will use this source to directly measure the

plasma/hot-gas cooling process and measure the absorption of injected laser pulses and possibly the heating of injected targets.

*This work performed under the auspices of the U.S. Department of Energy by Lawrence Livermore National Laboratory under Contract DE-AC52-07NA27344.

\section{REFERENCES}

[1] Moses, E.I., Diaz de la Rubia, T., Storm, E., Latkowski, J.F., Farmer, J.C., Abbott, R.P., Kramer, K.J., Peterson, P.F., Shaw, H.F., Lehman, R.F., “A Sustainable Nuclear Fuel Cycle Based on Laser Inertial Fusion Energy,” Fus. Sci. and Tech. 56, 547-565 (2009).

[2] HYADES, Cascade Applied Sciences, Longmont, CO 80503.

[3] MAXWELL, Ansoft, Pittsburgh, PA 15219.

[4] PSPICE, Cadence, San Jose, CA 95134.

[5] MECHANICAL, Ansys, Canonsburg, PA 15317

[6] J. O. Kane, M. A. Rhodes, G. A. Loosmore, J. F. Latkowski, J. M. Koning, M. V. Patel, H. A. Scott, G. B. Zimmerman, J. A. Demuth, G A. Moses, "Modeling of the LIFE Mini-chamber Xe theta pinch experiment," Photonics West LASE, SPIE paper 7916-5, 2011. 